\title{
Circadian and Seasonal Changes in Honeybee (Apis Mellifera) Worker Susceptibility to Pyrethroids
}

\section{Bartosz Piechowicz ${ }^{1 *}$, Przemysław Grodzicki ${ }^{2}$, Kinga Stawarczyk ${ }^{3}$, Iwona Piechowicz ${ }^{4}$, Michał Stawarczyk ${ }^{1}$, Aneta Zwolak ${ }^{1}$}

\author{
${ }^{1}$ Institute of Applied Biotechnology and Basic Science, Department of Ecotoxicology, University of Rzeszow, \\ Werynia 502, 36-100 Kolbuszowa, Poland \\ ${ }^{2}$ Faculty of Biology and Environmental Protection, Department of Animal Physiology, Nicolaus Copernicus University \\ Lwowska 1, 87-100 Toruń, Poland \\ ${ }^{3}$ Institute of Applied Biotechnology and Basic Science, Department of Botany, University of Rzeszow \\ Werynia 502, 36-100 Kolbuszowa, Poland \\ ${ }^{4}$ Communal Office in Niwiska, Niwiska 430, 36-147 Niwiska, Poland
}

Received: 28 November 2015

Accepted: 31 January 2016

\begin{abstract}
We examined the susceptibility of the worker honeybee Apis mellifera $\mathrm{L}$. to pyrethroids. Bees were intoxicated by selected synthetic pyrethroids (active substances: beta-cyfluthrin, deltamethrin, alphacypermethrin, lambda-cyhalothrin, esfenvalerate, and bifenthrin) and 72 hours after intoxication the number of surviving insects was determined. It was observed that the susceptibility of the honeybee to pyrethroids (expressed herein as the survival rate) was changing in diurnal rhythm, ranging from $100 \%$ (for a majority of substances in both seasons) to $11.1 \pm 0.37 \%$ (deltamethrin) and $11.1 \pm 0.73 \%$ (esfenvalerate) in spring, and $11.1 \pm 0.73 \%$ (esfenvalerate) and $4.6 \pm 0.17 \%$ (bifenthrin) in summer. The lowest susceptibility of bees to pyrethroids occurred during the night, when the honeybees were characterized by reduced motility and did not fly out for foraging, whereas the highest susceptibility was observed during the day, a natural time for seeking food. Along with diurnal changes in the susceptibility described above, differences dependent on the seasons in which the intoxication took place were also observed. Insects intoxicated in summer were less susceptible to pyrethroids than those intoxicated in spring.
\end{abstract}

Keywords: pyrethroids, insecticides, honeybee, biological rhythms, Apis mellifera

\section{Introduction}

Among many probable reasons for the occurrence of colony collapse disorder [1-7], one of the most important

*e-mail: bpiechow@univ.rzeszow.pl is the application of synthetic insecticides [8]. More than 100 insecticide substances are known whose toxicity to bees has been proven. The toxicity of these substances, including pyrethroids, is very often determined by their chemical structure. They are the most commonly used insecticides, utilized not only in agriculture, but also to 
destroy parasites in humans [9-11] and animals [12, 13], and household pests [14-16]. The harmful impact of these substances on bees has been described previously [17, 18]. The influence of pyrethroids involves, among other things, a modification of the kinetics of voltage-sensitive sodium channels [19-21], reduction in the AChE activity in the synaptic space [21], and, at the macro level, a reduction in colony size so that these chemicals act rapidly, causing the death of bees away from the hive. Pyrethroid preparations are characterised by a specific odour that may deter bees. This is believed to be a cause of their lower toxicity in field conditions compared to that which may be implied by the laboratory tests results [22].

The diurnal and seasonal changeability of the susceptibility to toxic compounds has been proved in many organisms [23], including honeybees [24, 25]. In the case of nectar-secreting plants, this calls for the recommendation to use the plant protection products at a time of the day when activity is reduced and, potentially, resistance against these substances increases. The issue seems to be important from the point of view of agricultural practices and environmental protection. When pesticides are used in the appropriate time of day, it is possible to reduce the application doses of plant protection products (PPPs) and their effectiveness would be adequate to eradicate pests.

The aim of the following research was to examine whether the susceptibility of $A$. mellifera to pyrethroid pesticides was related to the time of day and the season in which the insects were exposed to the preparation.

\section{Material and Methods}

Worker honeybees were collected directly at hive entrances in two apiaries located in Podleszany (2008) and Tarnobrzeg (2009) of Sub-Carpathian Province. According to the owners, colonies of bees from both apiaries during both seasons of research were genetically derived from the same source (the queens originated from the same breeding of queen bees). The intoxication of the insects took place at the turn of April and May (for spring measurement) and in July (for summer measurement). In total, 18,144 workers were used.

The aqueous solutions of the following insecticides from the group of pyrethroids were used:

- Bulldock 25 EC. Producer: Irvita Plant Protection N.V; active substance: beta-cyfluthrin $-25 \mathrm{~g}$ in 1 litre of the agent; concentration of usable liquid $4 \mathrm{mg} / \mathrm{L}$.

- Decis 005 UL. Producer: Bayer CropScience SA; active substance: deltamethrin $-5 \mathrm{~g}$ in 1 litre of the agent; concentration of usable liquid $3 \mathrm{mg} / \mathrm{L}$.

- Fastac 100 EC. Producer: BASF Agro B.V.; active substance: alpha-cypermethrin - $100 \mathrm{~g}$ in 1 litre of the agent; concentration of usable liquid $3 \mathrm{mg} / \mathrm{L}$.

- Karate-Zeon 050 CS. Producer: Syngenta Limited; active substance: lambda-cyhalothrin $-50 \mathrm{~g}$ in 1 litre of the agent; concentration of usable liquid $4 \mathrm{mg} / \mathrm{L}$.

- Sumi-Alpha 050 EC. Producer: Sumitomo Chemical Company Limited; active substance: esfenvalerate
$-50 \mathrm{~g}$ in 1 litre of the agent; concentration of usable liquid $2 \mathrm{mg} / \mathrm{L}$.

- Talstar 100 EC. Producer: FMC Corporation. Active substance: bifenthrin - $100 \mathrm{~g}$ in 1 litre of the agent; concentration of usable liquid $9 \mathrm{mg} / \mathrm{L}$.

Worker honeybees were placed in a modified incubator Q-Cell model ERC0750 in stable thermal conditions of $27^{\circ} \mathrm{C} \pm 0.5^{\circ} \mathrm{C}$, with the use of the natural $\mathrm{L} / \mathrm{D}$ cycle (an incubator with a glass window, placed in the utility room with the window oriented north to get a natural diurnal rhythm of light and darkness, and to prevent the exposure of bees to direct sunlight). The animals had unlimited access to food and water. Insects were taken from the hive entrance no later than two hours before the insecticide application. The contact apitoxicity was determined by an individual dosing method. An applicator was used to place a drop of the preparation $(4 \mu \mathrm{l})$ on the ventral part of the insect's thorax near the paraoesophageal ring. Water was used in the control group. Each test was performed on 108 individuals (18 individuals in 6 repetitions). The assumed duration of each measuring cycle was 72 hours. After this time, the surviving insects were counted. We chose to use 72-hour testing because pyrethroids are compounds that in animal tissues undergo rapid detoxification and, according to our previous studies on different insects using PPPs, a period of three days is critical as those insects that survived this period usually regained full health [24-27].

The results were developed using Statistica for Windows software v. 10 with the use of multivariate analysis of ANOVA (Tukey's test). The effect of the application time was evaluated using one-way ANOVA (Tables 2 and 4). Two-way ANOVA was used to determine the combined effect of the application time and the season (Table 5).

\section{Results}

In spring and summer bees from the control group showed higher survivability than those observed in the group treated with pyrethroids. The application of pyrethroids caused a significant decrease in the survivability of worker bees compared to the control group (Tables 1 and 3).

Regardless of the season and the preparation used, the susceptibility of bees to insecticides varied depending on the time of application. The highest susceptibility was exhibited by insects that were exposed to insecticides during the day (i.e., in the period of naturally raised activity of bees; Tables 1 and 3). In the experiments conducted in spring, the survivability rates achieved several times $100 \%$ during the night hours, decreasing at the diurnal peak of the insecticide action to $57.4 \%$ in insects treated with beta-cyfluthrin, and to only $11.1 \%$ in bees treated with deltamethrin and esfenvalerate (Table 1). Similarly, in summer the survivability of bees was reduced to $11.1 \%$ and $4.4 \%$ after the use of esfenvalerate and bifenthrin, respectively. At that time, in the worker honeybee groups treated with deltamethrin, esfenvalerate, and bifenthrin, no 
Table 1. The effects of insecticide intoxication with pesticides on survivability of honeybee (A. mellifera) workers in spring. A - control, $\mathrm{B}$ - beta-cyfluthrin, C - delamethrin, D - alpha-cypermethrin, E - lambda-cyhalothrin, F - esfenvalerate, G - bifenthrin.

\begin{tabular}{|c|c|c|c|c|c|c|c|c|c|c|c|c|c|}
\hline $\begin{array}{l}\text { Active } \\
\text { subst }\end{array}$ & $\mathrm{n}=108$ & \multicolumn{12}{|c|}{ Time of application } \\
\hline & & 02:00 & 04:00 & 06:00 & 08:00 & $10: 00$ & $12: 00$ & $14: 00$ & $16: 00$ & 18:00 & $20: 00$ & $22: 00$ & 00:00 \\
\hline \multirow{2}{*}{ A } & survi. (\%) & 100.0 & 100.0 & 100.0 & 99.1 & 92.6 & 90.7 & 93.5 & 100.0 & 99.1 & 100.0 & 100.0 & 100.0 \\
\hline & std. err. & \pm 0.00 & \pm 0.00 & \pm 0.00 & \pm 0.17 & \pm 0.33 & \pm 0.21 & \pm 0.17 & \pm 0.00 & \pm 0.17 & \pm 0.00 & \pm 0.00 & \pm 0.00 \\
\hline \multirow{2}{*}{ B } & survi. (\%) & 100.0 & 100.0 & 85.2 & 92.6 & 90.7 & 71.3 & 59.3 & 57.4 & 83.3 & 80.6 & 93.5 & 87.0 \\
\hline & std. err. & \pm 0.00 & \pm 0.00 & \pm 0.42 & \pm 0.21 & \pm 0.21 & \pm 0.75 & \pm 0.33 & \pm 0.33 & \pm 0.45 & \pm 0.34 & \pm 0.17 & \pm 0.33 \\
\hline \multirow{2}{*}{$\mathrm{C}$} & survi. (\%) & 100.0 & 100.0 & 91.7 & 91.7 & 73.1 & 48.1 & 11.1 & 41.7 & 63.9 & 92.6 & 80.6 & 90.7 \\
\hline & std. err. & \pm 0.00 & \pm 0.00 & \pm 0.34 & \pm 0.22 & \pm 0.65 & \pm 0.21 & \pm 0.37 & \pm 0.34 & \pm 0.34 & \pm 0.33 & \pm 0.34 & \pm 0.33 \\
\hline \multirow{2}{*}{$\mathrm{D}$} & survi. (\%) & 100.0 & 100.0 & 100.0 & 93.5 & 93.5 & 72.2 & 55.6 & 61.1 & 83.3 & 88.0 & 100.0 & 94.4 \\
\hline & std. err. & \pm 0.00 & \pm 0.00 & \pm 0.00 & \pm 0.54 & \pm 0.54 & \pm 0.58 & \pm 0.63 & \pm 0.77 & \pm 0.52 & \pm 0.70 & \pm 0.00 & \pm 0.52 \\
\hline \multirow{2}{*}{$\mathrm{E}$} & survi. (\%) & 100.0 & 89.8 & 82.4 & 100.0 & 71.3 & 59.3 & 32.4 & 48.1 & 77.8 & 76.9 & 93.5 & 100.0 \\
\hline & std. err. & \pm 0.00 & \pm 0.17 & \pm 0.70 & \pm 0.00 & \pm 0.48 & \pm 0.33 & \pm 0.31 & \pm 0.49 & \pm 0.77 & \pm 0.70 & \pm 0.31 & \pm 0.00 \\
\hline \multirow{2}{*}{$\mathrm{F}$} & survi. (\%) & 100.0 & 100.0 & 100.0 & 92.6 & 49.1 & 16.7 & 11.1 & 56.5 & 82.4 & 92.6 & 100.0 & 92.6 \\
\hline & std. err. & \pm 0.00 & \pm 0.00 & \pm 0.00 & \pm 0.49 & \pm 0.40 & \pm 0.52 & \pm 0.73 & \pm .65 & \pm 0.48 & \pm 1.15 & \pm 0.00 & \pm 0.33 \\
\hline \multirow{2}{*}{ G } & survi. (\%) & 100.0 & 93.5 & 87.0 & 93.5 & 82.4 & 38.0 & 41.7 & 47.2 & 54.6 & 86.1 & 89.8 & 97.2 \\
\hline & std. err. & \pm 0.00 & \pm 0.17 & \pm 0.33 & \pm 0.31 & \pm 0.54 & \pm 0.83 & \pm 0.34 & \pm 0.22 & \pm 0.48 & \pm 0.34 & \pm 0.65 & \pm 0.50 \\
\hline
\end{tabular}

survivability at the level of $100 \%$ (Table 3 ) was noted in any case. A lot of differences observed in the survivability of insects in the 24-hour cycle proved to be statistically significant (Tables 2 and 4).
The analysis of seasonal changes in honeybee susceptibility to pyrethroids showed that, apart from the case of bees treated with beta-cyfluthrin, diurnal peaks of insecticide action in summer months occurred earlier

Table 2. The list of statistically significant differences in the survivability of the worker honeybees (A. mellifera) under the influence of pesticides in spring, dependent on the hour of intoxication. A - control, B - beta-cyfluthrin, C - delamethrin, D - alpha-cypermethrin, $\mathrm{E}$ - lambda-cyhalothrin, $\mathrm{F}$ - esfenvalerate, $\mathrm{G}$ - bifenthrin. ${ }^{1} \mathrm{P}<0.05,{ }^{2} \mathrm{P}<0.01,{ }^{3} \mathrm{P}<0.001$.

\begin{tabular}{|c|c|c|c|c|c|c|c|c|c|c|c|c|}
\hline & \multicolumn{12}{|c|}{ Time of application } \\
\hline $\begin{array}{l}\text { Active } \\
\text { subst. }\end{array}$ & 02:00 & 04:00 & 06:00 & 08:00 & $10: 00$ & $12: 00$ & $14: 00$ & $16: 00$ & $18: 00$ & $20: 00$ & $22: 00$ & 00:00 \\
\hline A & $\begin{array}{l}10: 00^{3} \\
12: 00^{3} \\
14: 00^{3}\end{array}$ & $\begin{array}{l}10: 00^{3} \\
12: 00^{3} \\
14: 00^{3}\end{array}$ & $\begin{array}{l}10: 00^{3} \\
12: 00^{3} \\
14: 00^{3}\end{array}$ & $\begin{array}{l}10: 00^{3} \\
12: 00^{3} \\
14: 00^{3}\end{array}$ & $\begin{array}{l}02: 00^{3} \\
04: 00^{3} \\
06: 00^{3} \\
08: 00^{3} \\
16: 00^{3} \\
18: 00^{3} \\
20: 00^{3} \\
22: 00^{3} \\
00: 00^{3}\end{array}$ & $\begin{array}{l}02: 00^{3} \\
04: 00^{3} \\
06: 00^{3} \\
08: 00^{3} \\
16: 00^{3} \\
18: 00^{3} \\
20: 00^{3} \\
22: 00^{3} \\
00: 00^{3}\end{array}$ & $\begin{array}{l}02: 00^{3} \\
04: 00^{3} \\
06: 00^{3} \\
08: 00^{3} \\
16: 00^{3} \\
18: 00^{3} \\
20: 00^{3} \\
22: 00^{3} \\
00: 00^{3}\end{array}$ & $\begin{array}{l}10: 00^{3} \\
12: 00^{3} \\
14: 00^{3}\end{array}$ & $\begin{array}{l}10: 00^{3} \\
12: 00^{3} \\
14: 00^{3}\end{array}$ & $\begin{array}{l}10: 00^{3} \\
12: 00^{3} \\
14: 00^{3}\end{array}$ & $\begin{array}{l}10: 00^{3} \\
12: 00^{3} \\
14: 00^{3}\end{array}$ & $\begin{array}{l}10: 00^{3} \\
12: 00^{3} \\
14: 00^{3}\end{array}$ \\
\hline B & $\begin{array}{l}06: 00^{3} \\
12: 00^{3} \\
14: 00^{3} \\
16: 00^{3} \\
18: 00^{3} \\
20: 00^{3} \\
00: 00^{2}\end{array}$ & $\begin{array}{l}06: 00^{3} \\
12: 00^{3} \\
14: 00^{3} \\
16: 00^{3} \\
18: 00^{3} \\
20: 00^{3} \\
00: 00^{2}\end{array}$ & $\begin{array}{l}02: 00^{3} \\
04: 00^{3} \\
12: 00^{3} \\
14: 00^{3} \\
16: 00^{3}\end{array}$ & $\begin{array}{l}12: 00^{3} \\
14: 00^{3} \\
16: 00^{3} \\
20: 00^{2}\end{array}$ & $\begin{array}{l}12: 00^{3} \\
14: 00^{3} \\
16: 00^{3} \\
20: 00^{1}\end{array}$ & $\begin{array}{l}02: 00^{3} \\
04: 00^{3} \\
06: 00^{3} \\
08: 00^{3} \\
10: 00^{3} \\
14: 00^{2} \\
16: 00^{3} \\
18: 00^{2} \\
22: 00^{3} \\
00: 00^{3}\end{array}$ & $\begin{array}{l}02: 00^{3} \\
04: 00^{3} \\
06: 00^{3} \\
08: 00^{3} \\
10: 00^{3} \\
12: 00^{2} \\
18: 00^{3} \\
20: 00^{3} \\
22: 00^{3} \\
00: 00^{3}\end{array}$ & $\begin{array}{l}02: 00^{3} \\
04: 00^{3} \\
06: 00^{3} \\
08: 00^{3} \\
10: 00^{3} \\
12: 00^{3} \\
18: 00^{3} \\
20: 00^{3} \\
22: 00^{3} \\
00: 00^{3}\end{array}$ & $\begin{array}{l}02: 00^{3} \\
04: 00^{3} \\
12: 00^{2} \\
14: 00^{3} \\
16: 00^{3} \\
22: 00^{1}\end{array}$ & $\begin{array}{l}02: 00^{3} \\
04: 00^{3} \\
08: 00^{2} \\
10: 00^{1} \\
14: 00^{3} \\
16: 00^{3} \\
22: 00^{2}\end{array}$ & $\begin{array}{l}12: 00^{3} \\
14: 00^{3} \\
16: 00^{3} \\
18: 00^{1} \\
20: 00^{2}\end{array}$ & $\begin{array}{l}02: 00^{2} \\
04: 00^{2} \\
12: 00^{3} \\
14: 00^{3} \\
16: 00^{3}\end{array}$ \\
\hline
\end{tabular}


Table 2. Continued.

\begin{tabular}{|c|c|c|c|c|c|c|c|c|c|c|c|c|}
\hline & \multicolumn{12}{|c|}{ Time of application } \\
\hline $\begin{array}{l}\text { Active } \\
\text { subst. }\end{array}$ & 02:00 & 04:00 & 06:00 & 08:00 & $10: 00$ & $12: 00$ & $14: 00$ & $16: 00$ & $18: 00$ & $20: 00$ & $22: 00$ & 00:00 \\
\hline $\mathrm{C}$ & $\begin{array}{l}10: 00^{3} \\
12: 00^{3} \\
14: 00^{3} \\
16: 00^{3} \\
18: 00^{3} \\
22: 00^{3} \\
00: 00^{1}\end{array}$ & $\begin{array}{l}10: 00^{3} \\
12: 00^{3} \\
14: 00^{3} \\
16: 00^{3} \\
18: 00^{3} \\
22: 00^{3} \\
00: 00^{1}\end{array}$ & $\begin{array}{l}10: 00^{3} \\
12: 00^{3} \\
14: 00^{3} \\
16: 00^{3} \\
18: 00^{3} \\
22: 00^{2}\end{array}$ & $\begin{array}{l}10: 00^{3} \\
12: 00^{3} \\
14: 00^{3} \\
16: 00^{3} \\
18: 00^{3} \\
22: 00^{2}\end{array}$ & $\begin{array}{l}02: 00^{3} \\
04: 00^{3} \\
06: 00^{3} \\
08: 00^{3} \\
12: 00^{3} \\
14: 00^{3} \\
16: 00^{3} \\
18: 00^{1} \\
20: 00^{3} \\
00: 00^{3}\end{array}$ & $\begin{array}{l}02: 00^{3} \\
04: 00^{3} \\
06: 00^{3} \\
08: 00^{3} \\
10: 00^{3} \\
14: 00^{3} \\
18: 00^{3} \\
20: 00^{3} \\
22: 00^{3} \\
00: 00^{3}\end{array}$ & $\begin{array}{l}02: 00^{3} \\
04: 00^{3} \\
06: 00^{3} \\
08: 00^{3} \\
10: 00^{3} \\
12: 00^{3} \\
16: 00^{3} \\
18: 00^{3} \\
20: 00^{3} \\
22: 00^{3} \\
00: 00^{3}\end{array}$ & $\begin{array}{c}02: 00^{3} \\
04: 00^{3} \\
06: 00^{3} \\
08: 00^{3} \\
10: 00^{3} \\
14: 00^{3} \\
18: 00^{3} \\
20: 00^{3} \\
22: 00^{3} \\
00: 00^{3}\end{array}$ & $\begin{array}{l}02: 00^{3} \\
04: 00^{3} \\
06: 00^{3} \\
08: 00^{3} \\
10: 00^{1} \\
12: 00^{3} \\
14: 00^{3} \\
16: 00^{3} \\
20: 00^{3} \\
22: 00^{3} \\
00: 00^{3}\end{array}$ & $\begin{array}{l}10: 00^{3} \\
12: 00^{3} \\
14: 00^{3} \\
16: 00^{3} \\
18: 00^{3} \\
22: 00^{3}\end{array}$ & $\begin{array}{l}02: 00^{3} \\
04: 00^{3} \\
06: 00^{2} \\
08: 00^{2} \\
12: 00^{3} \\
14: 00^{3} \\
16: 00^{3} \\
18: 00^{3} \\
20: 00^{2} \\
00: 00^{1}\end{array}$ & $\begin{array}{l}02: 00^{1} \\
04: 00^{1} \\
10: 00^{3} \\
12: 00^{3} \\
14: 00^{3} \\
16: 00^{3} \\
18: 00^{3} \\
22: 00^{1}\end{array}$ \\
\hline $\mathrm{D}$ & $\begin{array}{l}12: 00^{3} \\
14: 00^{3} \\
16: 00^{3} \\
18: 00^{2}\end{array}$ & $\begin{array}{l}12: 00^{3} \\
14: 00^{3} \\
16: 00^{3} \\
18: 00^{2}\end{array}$ & $\begin{array}{l}12: 00^{3} \\
14: 00^{3} \\
16: 00^{3} \\
18: 00^{2}\end{array}$ & $\begin{array}{l}12: 00^{3} \\
14: 00^{3} \\
16: 00^{3}\end{array}$ & $\begin{array}{l}12: 00^{3} \\
14: 00^{3} \\
16: 00^{3}\end{array}$ & $\begin{array}{l}02: 00^{3} \\
04: 00^{3} \\
06: 00^{3} \\
08: 00^{3} \\
10: 00^{3} \\
14: 00^{2} \\
20: 00^{2} \\
22: 00^{3} \\
00: 00^{3}\end{array}$ & $\begin{array}{l}02: 00^{3} \\
04: 00^{3} \\
06: 00^{3} \\
08: 00^{3} \\
10: 00^{3} \\
12: 00^{2} \\
18: 00^{3} \\
20: 00^{3} \\
22: 00^{3} \\
00: 00^{3}\end{array}$ & $\begin{array}{l}02: 00^{3} \\
04: 00^{3} \\
06: 00^{3} \\
08: 00^{3} \\
10: 00^{3} \\
18: 00^{3} \\
20: 00^{3} \\
22: 00^{3} \\
00: 00^{3}\end{array}$ & $\begin{array}{l}02: 00^{2} \\
04: 00^{2} \\
06: 00^{2} \\
14: 00^{3} \\
16: 00^{3} \\
22: 00^{2}\end{array}$ & $\begin{array}{l}12: 00^{2} \\
14: 00^{3} \\
16: 00^{3}\end{array}$ & $\begin{array}{l}12: 00^{3} \\
14: 00^{3} \\
16: 00^{3} \\
18: 00^{2}\end{array}$ & $\begin{array}{l}12: 00^{3} \\
14: 00^{3} \\
16: 00^{3}\end{array}$ \\
\hline $\mathrm{E}$ & $\begin{array}{l}06: 00^{3} \\
10: 00^{3} \\
12: 00^{3} \\
14: 00^{3} \\
16: 00^{3} \\
18: 00^{3} \\
20: 00^{3}\end{array}$ & $\begin{array}{l}10: 00^{3} \\
12: 00^{3} \\
14: 00^{3} \\
16: 00^{3} \\
18: 00^{1} \\
20: 00^{1}\end{array}$ & $\begin{array}{l}02: 00^{3} \\
08: 00^{3} \\
12: 00^{3} \\
14: 00^{3} \\
16: 00^{3} \\
00: 00^{3}\end{array}$ & $\begin{array}{l}06: 00^{3} \\
10: 00^{3} \\
12: 00^{3} \\
14: 00^{3} \\
16: 00^{3} \\
18: 00^{3} \\
20: 00^{3}\end{array}$ & $\begin{array}{l}02: 00^{3} \\
04: 00^{3} \\
08: 00^{3} \\
12: 00^{1} \\
14: 00^{3} \\
16: 00^{3} \\
22: 00^{3} \\
00: 00^{3}\end{array}$ & $\begin{array}{l}02: 00^{3} \\
04: 00^{3} \\
06: 00^{3} \\
08: 00^{3} \\
10: 00^{1} \\
14: 00^{3} \\
18: 00^{3} \\
20: 00^{3} \\
22: 00^{3} \\
00: 00^{3}\end{array}$ & $\begin{array}{l}02: 00^{3} \\
04: 00^{3} \\
06: 00^{3} \\
08: 00^{3} \\
10: 00^{3} \\
12: 00^{3} \\
16: 00^{3} \\
18: 00^{3} \\
20: 00^{3} \\
22: 00^{3} \\
00: 00^{3}\end{array}$ & $\begin{array}{c}02: 00^{3} \\
04: 00^{3} \\
06: 00^{3} \\
08: 00^{3} \\
10: 00^{3} \\
14: 00^{2} \\
18: 00^{3} \\
20: 00^{3} \\
22: 00^{3} \\
00: 00^{3}\end{array}$ & $\begin{array}{l}02: 00^{3} \\
04: 00^{1} \\
08: 00^{3} \\
12: 00^{3} \\
14: 00^{3} \\
16: 00^{3} \\
22: 00^{2} \\
00: 00^{3}\end{array}$ & $\begin{array}{l}02: 00^{3} \\
04: 00^{1} \\
08: 00^{3} \\
12: 00^{3} \\
14: 00^{3} \\
16: 00^{3} \\
22: 00^{3} \\
00: 00^{3}\end{array}$ & $\begin{array}{l}10: 00^{3} \\
12: 00^{3} \\
14: 00^{3} \\
16: 00^{3} \\
18: 00^{2} \\
20: 00^{3}\end{array}$ & $\begin{array}{l}06: 00^{3} \\
10: 00^{3} \\
12: 00^{3} \\
14: 00^{3} \\
16: 00^{3} \\
18: 00^{3} \\
20: 00^{3}\end{array}$ \\
\hline $\mathrm{F}$ & $\begin{array}{l}10: 00^{3} \\
12: 00^{3} \\
14: 00^{3} \\
16: 00^{3} \\
18: 00^{2}\end{array}$ & $\begin{array}{l}10: 00^{3} \\
12: 00^{3} \\
14: 00^{3} \\
16: 00^{3} \\
18: 00^{2}\end{array}$ & $\begin{array}{l}10: 00^{3} \\
12: 00^{3} \\
14: 00^{3} \\
16: 00^{3} \\
18: 00^{2}\end{array}$ & $\begin{array}{l}10: 00^{3} \\
12: 00^{3} \\
14: 00^{3} \\
16: 00^{3}\end{array}$ & $\begin{array}{l}02: 00^{3} \\
04: 00^{3} \\
06: 00^{3} \\
08: 00^{3} \\
12: 00^{3} \\
14: 00^{3} \\
18: 00^{3} \\
20: 00^{3} \\
22: 00^{3} \\
00: 00^{3}\end{array}$ & $\begin{array}{l}02: 00^{3} \\
04: 00^{3} \\
06: 00^{3} \\
08: 00^{3} \\
10: 00^{3} \\
16: 00^{3} \\
18: 00^{3} \\
20: 00^{3} \\
22: 00^{3} \\
00: 00^{3}\end{array}$ & $\begin{array}{c}02: 00^{3} \\
04: 00^{3} \\
06: 00^{3} \\
08: 00^{3} \\
10: 00^{3} \\
16: 00^{3} \\
18: 00^{3} \\
20: 00^{3} \\
22: 00^{3} \\
00: 00^{3}\end{array}$ & $\begin{array}{c}02: 00^{3} \\
04: 00^{3} \\
06: 00^{3} \\
08: 00^{3} \\
12: 00^{3} \\
14: 00^{3} \\
18: 00^{3} \\
20: 00^{3} \\
22: 00^{3} \\
00: 00^{3}\end{array}$ & $\begin{array}{l}02: 00^{2} \\
04: 00^{2} \\
06: 00^{2} \\
10: 00^{3} \\
12: 00^{3} \\
14: 00^{3} \\
16: 00^{3} \\
22: 00^{2}\end{array}$ & $\begin{array}{l}10: 00^{3} \\
12: 00^{3} \\
14: 00^{3} \\
16: 00^{3}\end{array}$ & $\begin{array}{l}10: 00^{3} \\
12: 00^{3} \\
14: 00^{3} \\
16: 00^{3} \\
18: 00^{2}\end{array}$ & $\begin{array}{l}10: 00^{3} \\
12: 00^{3} \\
14: 00^{3} \\
16: 00^{3}\end{array}$ \\
\hline G & $\begin{array}{l}06: 00^{1} \\
10: 00^{3} \\
12: 00^{3} \\
14: 00^{3} \\
16: 00^{3} \\
18: 00^{3} \\
20: 00^{1}\end{array}$ & $\begin{array}{l}12: 00^{3} \\
14: 00^{3} \\
16: 00^{3} \\
18: 00^{3}\end{array}$ & $\begin{array}{l}02: 00^{1} \\
12: 00^{3} \\
14: 00^{3} \\
16: 00^{3} \\
18: 00^{3}\end{array}$ & $\begin{array}{l}12: 00^{3} \\
14: 00^{3} \\
16: 00^{3} \\
18: 00^{3}\end{array}$ & $\begin{array}{l}02: 00^{3} \\
12: 00^{3} \\
14: 00^{3} \\
16: 00^{3} \\
18: 00^{3} \\
00: 00^{2}\end{array}$ & $\begin{array}{l}02: 00^{3} \\
04: 00^{3} \\
06: 00^{3} \\
08: 00^{3} \\
10: 00^{3} \\
18: 00^{3} \\
20: 00^{3} \\
22: 00^{3} \\
00: 00^{3}\end{array}$ & $\begin{array}{l}02: 00^{3} \\
04: 00^{3} \\
06: 00^{3} \\
08: 00^{3} \\
10: 00^{3} \\
18: 00^{1} \\
20: 00^{3} \\
22: 00^{3} \\
00: 00^{3}\end{array}$ & $\begin{array}{l}02: 00^{3} \\
04: 00^{3} \\
06: 00^{3} \\
08: 00^{3} \\
10: 00^{3} \\
20: 00^{3} \\
22: 00^{3} \\
00: 00^{3}\end{array}$ & $\begin{array}{c}02: 00^{3} \\
04: 00^{3} \\
06: 00^{3} \\
08: 00^{3} \\
10: 00^{3} \\
12: 00^{3} \\
14: 00^{1} \\
20: 00^{3} \\
22: 00^{3} \\
00: 00^{3}\end{array}$ & $\begin{array}{l}02: 00^{1} \\
12: 00^{3} \\
14: 00^{3} \\
16: 00^{3} \\
18: 00^{3}\end{array}$ & $\begin{array}{l}12: 00^{3} \\
14: 00^{3} \\
16: 00^{3} \\
18: 00^{3}\end{array}$ & $\begin{array}{l}10: 00^{2} \\
12: 00^{3} \\
14: 00^{3} \\
16: 00^{3} \\
18: 00^{3}\end{array}$ \\
\hline
\end{tabular}


Table 3. The effect of insecticide intoxication with pesticides on survivability of honeybee (A. mellifera) workers in summer. A - control, $\mathrm{B}$ - beta-cyfluthrin, C - delamethrin, D - alpha-cypermethrin, E - lambda-cyhalothrin, F - esfenvalerate, G - bifenthrin.

\begin{tabular}{|c|c|c|c|c|c|c|c|c|c|c|c|c|c|}
\hline Active & $\mathrm{n}=108$ & \multicolumn{12}{|c|}{ Time of application } \\
\hline & & 02:00 & 04:00 & 06:00 & 08:00 & $10: 00$ & $12: 00$ & $14: 00$ & $16: 00$ & $18: 00$ & $20: 00$ & $22: 00$ & 00:00 \\
\hline \multirow{2}{*}{ A } & survi. (\%) & 100.0 & 91.7 & 100.0 & 100.0 & 95.4 & 94.4 & 95.4 & 100.0 & 99.1 & 100.0 & 91.7 & 100.0 \\
\hline & std. err. & \pm 0.00 & \pm 0.22 & \pm 0.00 & \pm 0.00 & \pm 0.17 & \pm 0.45 & \pm 0.31 & \pm 0.00 & \pm 0.17 & \pm 0.00 & \pm 0.22 & \pm 0.00 \\
\hline \multirow{2}{*}{ B } & survi. (\%) & 79.6 & 79.6 & 76.9 & 62.0 & 73.1 & 68.5 & 37.0 & 66.7 & 22.2 & 100.0 & 87.0 & 92.6 \\
\hline & std. err. & \pm 0.21 & \pm 0.33 & \pm 0.60 & \pm 0.40 & \pm 0.79 & \pm 0.33 & \pm 0.80 & \pm 0.68 & \pm 0.26 & \pm 0.00 & \pm 0.33 & \pm 0.61 \\
\hline \multirow{2}{*}{$\mathrm{C}$} & survi. (\%) & 84.3 & 84.3 & 72.2 & 38.9 & 44.4 & 27.8 & 50.0 & 72.2 & 66.7 & 83.3 & 80.6 & 84.3 \\
\hline & std. err. & \pm 0.60 & \pm 0.65 & \pm 0.52 & \pm 0.68 & \pm 0.68 & \pm 0.45 & \pm 0.52 & \pm 0.58 & \pm 0.37 & \pm 0.52 & \pm 0.34 & \pm 0.65 \\
\hline \multirow{2}{*}{ D } & survi. (\%) & 100.0 & 82.4 & 94.4 & 54.6 & 78.7 & 16.7 & 50.9 & 76.9 & 88.9 & 93.5 & 94.4 & 100.0 \\
\hline & std. err. & \pm 0.00 & \pm 0.40 & \pm 0.45 & \pm 0.70 & \pm 0.31 & \pm 0.68 & \pm 0.40 & \pm 0.54 & \pm 0.45 & \pm 0.40 & \pm 0.45 & \pm 0.00 \\
\hline \multirow{2}{*}{ E } & survi. (\%) & 92.6 & 67.6 & 28.7 & 21.3 & 64.8 & 54.6 & 71.3 & 76.9 & 76.9 & 89.8 & 87.0 & 100.0 \\
\hline & std. err. & \pm 0.33 & \pm 0.79 & \pm 0.40 & \pm 0.79 & \pm 0.49 & \pm 0.70 & \pm 0.54 & \pm 0.65 & \pm 0.60 & \pm 0.17 & \pm 0.33 & \pm 0.00 \\
\hline \multirow{2}{*}{$\mathrm{F}$} & survi. (\%) & 88.9 & 92.6 & 81.5 & 11.1 & 17.6 & 37.0 & 89.8 & 93.5 & 100.0 & 84.3 & 93.5 & 93.5 \\
\hline & std. err. & \pm 0.26 & \pm 0.56 & \pm 0.92 & \pm 0.73 & \pm 0.40 & \pm 0.61 & \pm 0.40 & \pm 0.40 & \pm 0.00 & \pm 0.60 & \pm 0.48 & \pm 0.17 \\
\hline \multirow{2}{*}{ G } & survi. (\%) & 86.1 & 74.1 & 4.6 & 5.6 & 15.7 & 37.0 & 75.0 & 63.9 & 88.9 & 91.7 & 81.5 & 90.7 \\
\hline & std. err. & \pm 0.22 & \pm 0.49 & \pm 0.17 & \pm 0.63 & \pm 0.48 & \pm 0.67 & \pm 0.34 & \pm 0.22 & \pm 0.52 & \pm 0.34 & \pm 0.33 & \pm 0.33 \\
\hline
\end{tabular}

(deltamethrin, alpha-cypermethrin at 12:00; lambdacyhalothrin, esfenvalerate at 8:00; bifenthrin at 6:00; Table 3) than in spring months (deltamethrin, alphacypermethrin, lambda-cyhalothrin, esfenvalerate at 14:00; bifenthrin at 12:00; Tables 1, 3, and 5).

\section{Discussion}

The presence of cyclic changes in honeybee activity has been observed for nearly 100 years. With time passing, it has been proven that the age of the individuals, the

Table 4. The list of statistically significant differences in the survivability of worker honeybees (A. mellifera) under the influence of pesticides in summer. A - control, B - beta-cyfluthrin, C-delamethrin, D - alpha-cypermethrin, E-lambda-cyhalothrin, F-esfenvalerate, $\mathrm{G}-$ bifenthrin. ${ }^{1} \mathrm{P}<0.05,{ }^{2} \mathrm{P}<0.01,{ }^{3} \mathrm{P}<0.001$.

\begin{tabular}{|c|c|c|c|c|c|c|c|c|c|c|c|c|}
\hline & \multicolumn{12}{|c|}{ Time of application } \\
\hline $\begin{array}{l}\text { Active } \\
\text { subst. }\end{array}$ & 02:00 & 04:00 & 06:00 & 08:00 & $10: 00$ & $12: 00$ & $14: 00$ & $16: 00$ & $18: 00$ & $20: 00$ & $22: 00$ & 00:00 \\
\hline A & $\begin{array}{l}04: 00^{3} \\
12: 00^{1} \\
22: 00^{3}\end{array}$ & $\begin{array}{l}02: 00^{3} \\
06: 00^{3} \\
08: 00^{3} \\
16: 00^{3} \\
18: 00^{3} \\
20: 00^{3} \\
00: 00^{3}\end{array}$ & $\begin{array}{l}04: 00^{3} \\
12: 00^{1} \\
22: 00^{3}\end{array}$ & $\begin{array}{l}04: 00^{3} \\
12: 00^{1} \\
22: 00^{3}\end{array}$ & - & $\begin{array}{l}02: 00^{1} \\
06: 00^{1} \\
08: 00^{1} \\
16: 00^{1} \\
20: 00^{1} \\
00: 00^{1}\end{array}$ & - & $\begin{array}{l}04: 00^{3} \\
12: 00^{1} \\
22: 00^{3}\end{array}$ & $\begin{array}{l}04: 00^{3} \\
22: 00^{3}\end{array}$ & $\begin{array}{l}04: 00^{3} \\
12: 00^{1} \\
22: 00^{3}\end{array}$ & $\begin{array}{l}02: 00^{3} \\
06: 00^{3} \\
08: 00^{3} \\
16: 00^{3} \\
18: 00^{3} \\
20: 00^{3} \\
00: 00^{3}\end{array}$ & $\begin{array}{l}04: 00^{3} \\
12: 00^{1} \\
22: 00^{3}\end{array}$ \\
\hline B & $\begin{array}{l}08: 00^{2} \\
14: 00^{3} \\
18: 00^{3} \\
20: 00^{3}\end{array}$ & $\begin{array}{l}08: 00^{2} \\
14: 00^{3} \\
18: 00^{3} \\
20: 00^{3}\end{array}$ & $\begin{array}{l}08: 00^{1} \\
14: 00^{3} \\
18: 00^{3} \\
20: 00^{3} \\
00: 00^{1}\end{array}$ & $\begin{array}{l}02: 00^{2} \\
04: 00^{2} \\
06: 00^{1} \\
14: 00^{3} \\
18: 00^{3} \\
20: 00^{3} \\
22: 00^{3} \\
00: 00^{3}\end{array}$ & $\begin{array}{l}14: 00^{3} \\
18: 00^{3} \\
20: 00^{3} \\
22: 00^{1} \\
00: 00^{3}\end{array}$ & $\begin{array}{l}14: 00^{3} \\
18: 00^{3} \\
20: 00^{3} \\
22: 00^{2} \\
00: 00^{3}\end{array}$ & $\begin{array}{l}02: 00^{3} \\
04: 00^{3} \\
06: 00^{3} \\
08: 00^{3} \\
10: 00^{3} \\
12: 00^{3} \\
16: 00^{3} \\
18: 00^{1} \\
20: 00^{3} \\
22: 00^{3} \\
00: 00^{3}\end{array}$ & $\begin{array}{l}14: 00^{3} \\
18: 00^{3} \\
20: 00^{3} \\
22: 00^{3} \\
00: 00^{3}\end{array}$ & $\begin{array}{l}02: 00^{3} \\
04: 00^{3} \\
06: 00^{3} \\
08: 00^{3} \\
10: 00^{3} \\
12: 00^{3} \\
14: 00^{1} \\
16: 00^{3} \\
20: 00^{3} \\
22: 00^{3} \\
00: 00^{3}\end{array}$ & $\begin{array}{l}02: 00^{3} \\
04: 00^{3} \\
06: 00^{3} \\
08: 00^{3} \\
10: 00^{3} \\
12: 00^{3} \\
14: 00^{3} \\
16: 00^{3} \\
18: 00^{3}\end{array}$ & $\begin{array}{l}08: 00^{3} \\
10: 00^{1} \\
12: 00^{2} \\
14: 00^{3} \\
16: 00^{3} \\
18: 00^{3}\end{array}$ & $\begin{array}{c}06: 00^{1} \\
08: 00^{3} \\
10: 00^{3} \\
12: 00^{3} \\
14: 00^{3} \\
16: 00^{3} \\
18: 00^{3}\end{array}$ \\
\hline
\end{tabular}


Table 4. Continued.

\begin{tabular}{|c|c|c|c|c|c|c|c|c|c|c|c|c|}
\hline & \multicolumn{12}{|c|}{ Time of application } \\
\hline $\begin{array}{l}\text { Active } \\
\text { subst. }\end{array}$ & 02:00 & 04:00 & 06:00 & 08:00 & $10: 00$ & $12: 00$ & $14: 00$ & $16: 00$ & 18:00 & 20:00 & $22: 00$ & 00:00 \\
\hline $\mathrm{C}$ & $\begin{array}{l}08: 00^{3} \\
10: 00^{3} \\
12: 00^{3} \\
14: 00^{3} \\
18: 00^{2}\end{array}$ & $\begin{array}{l}08: 00^{3} \\
10: 00^{3} \\
12: 00^{3} \\
14: 00^{3} \\
18: 00^{2}\end{array}$ & $\begin{array}{l}08: 00^{3} \\
10: 00^{3} \\
12: 00^{3} \\
14: 00^{3}\end{array}$ & $\begin{array}{l}02: 00^{3} \\
04: 00^{3} \\
06: 00^{3} \\
16: 00^{3} \\
18: 00^{3} \\
20: 00^{3} \\
22: 00^{3} \\
00: 00^{3}\end{array}$ & $\begin{array}{l}02: 00^{3} \\
04: 00^{3} \\
06: 00^{3} \\
12: 00^{1} \\
16: 00^{3} \\
18: 00^{3} \\
20: 00^{3} \\
22: 00^{3} \\
00: 00^{3}\end{array}$ & $\begin{array}{l}02: 00^{3} \\
04: 00^{3} \\
06: 00^{3} \\
10: 00^{1} \\
16: 00^{3} \\
18: 00^{3} \\
20: 00^{3} \\
22: 00^{3} \\
00: 00^{3}\end{array}$ & $\begin{array}{l}02: 00^{3} \\
04: 00^{3} \\
06: 00^{3} \\
12: 00^{3} \\
16: 00^{3} \\
18: 00^{1} \\
20: 00^{3} \\
22: 00^{3} \\
00: 00^{3}\end{array}$ & $\begin{array}{l}08: 00^{3} \\
10: 00^{3} \\
12: 00^{3} \\
14: 00^{3}\end{array}$ & $\begin{array}{l}02: 00^{2} \\
04: 00^{2} \\
08: 00^{3} \\
10: 00^{3} \\
12: 00^{3} \\
14: 00^{1} \\
20: 00^{1} \\
00: 00^{2}\end{array}$ & $\begin{array}{l}08: 00^{3} \\
10: 00^{3} \\
12: 00^{3} \\
14: 00^{3} \\
18: 00^{1}\end{array}$ & $\begin{array}{l}08: 00^{3} \\
10: 00^{3} \\
12: 00^{3} \\
14: 00^{3}\end{array}$ & $\begin{array}{l}08: 00^{3} \\
10: 00^{3} \\
12: 00^{3} \\
14: 00^{3} \\
18: 00^{2}\end{array}$ \\
\hline $\mathrm{D}$ & $\begin{array}{l}04: 00^{3} \\
08: 00^{3} \\
10: 00^{3} \\
12: 00^{3} \\
14: 00^{3} \\
16: 00^{3}\end{array}$ & $\begin{array}{c}02: 00^{3} \\
06: 00^{1} \\
08: 00^{3} \\
12: 00^{3} \\
14: 00^{3} \\
22: 00^{1} \\
00: 00^{3}\end{array}$ & $\begin{array}{l}04: 00^{1} \\
08: 00^{3} \\
10: 00^{2} \\
12: 00^{3} \\
14: 00^{3} \\
16: 00^{3}\end{array}$ & $\begin{array}{l}02: 00^{3} \\
04: 00^{3} \\
06: 00^{3} \\
10: 00^{3} \\
12: 00^{3} \\
16: 00^{3} \\
18: 00^{3} \\
20: 00^{3} \\
22: 00^{3} \\
00: 00^{3}\end{array}$ & $\begin{array}{l}02: 00^{3} \\
06: 00^{2} \\
08: 00^{3} \\
12: 00^{3} \\
14: 00^{3} \\
20: 00^{2} \\
22: 00^{2} \\
00: 00^{3}\end{array}$ & $\begin{array}{l}02: 00^{3} \\
04: 00^{3} \\
06: 00^{3} \\
08: 00^{3} \\
10: 00^{3} \\
14: 00^{3} \\
16: 00^{3} \\
18: 00^{3} \\
20: 00^{3} \\
22: 00^{3} \\
00: 00^{3}\end{array}$ & $\begin{array}{l}02: 00^{3} \\
04: 00^{3} \\
06: 00^{3} \\
10: 00^{3} \\
12: 00^{3} \\
16: 00^{3} \\
18: 00^{3} \\
20: 00^{3} \\
22: 00^{3} \\
00: 00^{3}\end{array}$ & $\begin{array}{c}02: 00^{3} \\
06: 00^{3} \\
08: 00^{3} \\
12: 00^{3} \\
14: 00^{3} \\
18: 00^{1} \\
20: 00^{3} \\
22: 00^{3} \\
00: 00^{3}\end{array}$ & $\begin{array}{l}08: 00^{3} \\
12: 00^{3} \\
14: 00^{3} \\
16: 00^{1}\end{array}$ & $\begin{array}{l}08: 00^{3} \\
10: 00^{2} \\
12: 00^{3} \\
14: 00^{3} \\
16: 00^{3}\end{array}$ & $\begin{array}{c}04: 00^{1} \\
08: 00^{3} \\
10: 00^{2} \\
12: 00^{3} \\
14: 00^{3} \\
16: 00^{3}\end{array}$ & $\begin{array}{l}04: 00^{3} \\
08: 00^{3} \\
10: 00^{3} \\
12: 00^{3} \\
14: 00^{3} \\
16: 00^{3}\end{array}$ \\
\hline $\mathrm{E}$ & $\begin{array}{c}04: 00^{3} \\
06: 00^{3} \\
08: 00^{3} \\
10: 00^{3} \\
12: 00^{3} \\
14: 00^{3} \\
16: 00^{1} \\
18: 00^{1}\end{array}$ & $\begin{array}{l}02: 00^{3} \\
06: 00^{3} \\
08: 00^{3} \\
20: 00^{3} \\
22: 00^{2} \\
00: 00^{3}\end{array}$ & $\begin{array}{l}02: 00^{3} \\
04: 00^{3} \\
10: 00^{3} \\
12: 00^{3} \\
14: 00^{3} \\
16: 00^{3} \\
18: 00^{3} \\
20: 00^{3} \\
22: 00^{3} \\
00: 00^{3}\end{array}$ & $\begin{array}{l}02: 00^{3} \\
04: 00^{3} \\
10: 00^{3} \\
12: 00^{3} \\
14: 00^{3} \\
16: 00^{3} \\
18: 00^{3} \\
20: 00^{3} \\
22: 00^{3} \\
00: 00^{3}\end{array}$ & $\begin{array}{l}02: 00^{3} \\
06: 00^{3} \\
08: 00^{3} \\
20: 00^{3} \\
22: 00^{3} \\
00: 00^{3}\end{array}$ & $\begin{array}{c}02: 00^{3} \\
06: 00^{3} \\
08: 00^{3} \\
14: 00^{1} \\
16: 00^{3} \\
18: 00^{3} \\
20: 00^{3} \\
22: 00^{3} \\
00: 00^{3}\end{array}$ & $\begin{array}{l}02: 00^{3} \\
06: 00^{3} \\
08: 00^{3} \\
12: 00^{1} \\
20: 00^{2} \\
22: 00^{1} \\
00: 00^{3}\end{array}$ & $\begin{array}{l}02: 00^{1} \\
06: 00^{3} \\
08: 00^{3} \\
12: 00^{3} \\
00: 00^{3}\end{array}$ & $\begin{array}{l}02: 00^{1} \\
06: 00^{3} \\
08: 00^{3} \\
12: 00^{3} \\
00: 00^{3}\end{array}$ & $\begin{array}{c}04: 00^{3} \\
06: 00^{3} \\
08: 00^{3} \\
10: 00^{3} \\
12: 00^{3} \\
14: 00^{2}\end{array}$ & $\begin{array}{c}04: 00^{2} \\
06: 00^{3} \\
08: 00^{3} \\
10: 00^{3} \\
12: 00^{3} \\
14: 00^{1}\end{array}$ & $\begin{array}{l}04: 00^{3} \\
06: 00^{3} \\
08: 00^{3} \\
10: 00^{3} \\
12: 00^{3} \\
14: 00^{3} \\
16: 00^{3} \\
18: 00^{3}\end{array}$ \\
\hline $\mathrm{F}$ & $\begin{array}{l}08: 00^{3} \\
10: 00^{3} \\
12: 00^{3}\end{array}$ & $\begin{array}{l}08: 00^{3} \\
10: 00^{3} \\
12: 00^{3}\end{array}$ & $\begin{array}{l}08: 00^{3} \\
10: 00^{3} \\
12: 00^{3} \\
18: 00^{2}\end{array}$ & $\begin{array}{l}02: 00^{3} \\
04: 00^{3} \\
06: 00^{3} \\
12: 00^{3} \\
14: 00^{3} \\
16: 00^{3} \\
18: 00^{3} \\
20: 00^{3} \\
22: 00^{3} \\
00: 00^{3}\end{array}$ & $\begin{array}{l}02: 00^{3} \\
04: 00^{3} \\
06: 00^{3} \\
12: 00^{3} \\
14: 00^{3} \\
16: 00^{3} \\
18: 00^{3} \\
20: 00^{3} \\
22: 00^{3} \\
00: 00^{3}\end{array}$ & $\begin{array}{l}02: 00^{3} \\
04: 00^{3} \\
06: 00^{3} \\
08: 00^{3} \\
10: 00^{3} \\
14: 00^{3} \\
16: 00^{3} \\
18: 00^{3} \\
20: 00^{3} \\
22: 00^{3} \\
00: 00^{3}\end{array}$ & $\begin{array}{l}08: 00^{3} \\
10: 00^{3} \\
12: 00^{3}\end{array}$ & $\begin{array}{l}08: 00^{3} \\
10: 00^{3} \\
12: 00^{3}\end{array}$ & $\begin{array}{l}06: 00^{2} \\
08: 00^{3} \\
10: 00^{3} \\
12: 00^{3} \\
20: 00^{1}\end{array}$ & $\begin{array}{l}08: 00^{3} \\
10: 00^{3} \\
12: 00^{3} \\
18: 00^{1}\end{array}$ & $\begin{array}{l}08: 00^{3} \\
10: 00^{3} \\
12: 00^{3}\end{array}$ & $\begin{array}{l}08: 00^{3} \\
10: 00^{3} \\
12: 00^{3}\end{array}$ \\
\hline G & $\begin{array}{c}04: 00^{1} \\
06: 00^{3} \\
08: 00^{3} \\
10: 00^{3} \\
12: 00^{3} \\
16: 00^{3}\end{array}$ & $\begin{array}{c}02: 00^{1} \\
06: 00^{3} \\
08: 00^{3} \\
10: 00^{3} \\
12: 00^{3} \\
18: 00^{2} \\
20: 00^{3} \\
00: 00^{3}\end{array}$ & $\begin{array}{l}02: 00^{3} \\
04: 00^{3} \\
12: 00^{3} \\
14: 00^{3} \\
16: 00^{3} \\
18: 00^{3} \\
20: 00^{3} \\
22: 00^{3} \\
00: 00^{3}\end{array}$ & $\begin{array}{l}02: 00^{3} \\
04: 00^{3} \\
12: 00^{3} \\
14: 00^{3} \\
16: 00^{3} \\
18: 00^{3} \\
20: 00^{3} \\
22: 00^{3} \\
00: 00^{3}\end{array}$ & $\begin{array}{l}02: 00^{3} \\
04: 00^{3} \\
12: 00^{3} \\
14: 00^{3} \\
16: 00^{3} \\
18: 00^{3} \\
20: 00^{3} \\
22: 00^{3} \\
00: 00^{3}\end{array}$ & $\begin{array}{l}02: 00^{3} \\
04: 00^{3} \\
06: 00^{3} \\
08: 00^{3} \\
10: 00^{3} \\
14: 00^{3} \\
16: 00^{3} \\
18: 00^{3} \\
20: 00^{3} \\
22: 00^{3} \\
00: 00^{3}\end{array}$ & $\begin{array}{l}06: 00^{3} \\
08: 00^{3} \\
10: 00^{3} \\
12: 00^{3} \\
18: 00^{2} \\
20: 00^{3} \\
00: 00^{3}\end{array}$ & $\begin{array}{c}02: 00^{3} \\
06: 00^{3} \\
08: 00^{3} \\
10: 00^{3} \\
12: 00^{3} \\
18: 00^{3} \\
20: 00^{3} \\
22: 00^{3} \\
00: 00^{3}\end{array}$ & $\begin{array}{c}04: 00^{2} \\
06: 00^{3} \\
08: 00^{3} \\
10: 00^{3} \\
12: 00^{3} \\
14: 00^{2} \\
16: 00^{3}\end{array}$ & $\begin{array}{c}04: 00^{3} \\
06: 00^{3} \\
08: 00^{3} \\
10: 00^{3} \\
12: 00^{3} \\
14: 00^{3} \\
16: 00^{3}\end{array}$ & $\begin{array}{l}06: 00^{3} \\
08: 00^{3} \\
10: 00^{3} \\
12: 00^{3} \\
16: 00^{3}\end{array}$ & $\begin{array}{c}04: 00^{3} \\
06: 00^{3} \\
08: 00^{3} \\
10: 00^{3} \\
12: 00^{3} \\
14: 00^{3} \\
16: 00^{3}\end{array}$ \\
\hline
\end{tabular}


Table 5. The list of statistically significant seasonal differences in the survivability of worker honeybees (A. mellifera), dependent on the influence of pesticides. A - control, B - beta-cyfluthrin, C - delamethrin, D - alpha-cypermethrin, E - lambda-cyhalothrin, $\mathrm{F}$ - esfenvalerate, $\mathrm{G}-$ bifenthrin. ${ }^{1} \mathrm{P}<0.05,{ }^{2} \mathrm{P}<0.01,{ }^{3} \mathrm{P}<0.001$.

$\left.\begin{array}{|c|c|}\hline \begin{array}{c}\text { Active } \\ \text { Subst. }\end{array} & \text { Time of application } \\ \hline \text { A } & 04: 00^{3} ; 22: 00^{3} \\ \hline \text { B } & 02: 00^{3} ; 04: 00^{3} ; 08: 00^{3} ; 10: 00^{3} ; 14: 00^{3} ; 18: 00^{3} ; \\ 20: 00^{3}\end{array}\right)$

functions they fulfil in the nest [28], the synchronization with other individuals in the colony [28-30], and the time of gathering nectar from plants [31] have the largest effect on the functioning of these insects' biological clocks.

In this study used individuals that had already done their cleansing flights. This meant that the bees showed a distinct 24-hour rhythm of activity. Rodriguez-Zas [28] gives examples of transcripts in the bodies of worker foragers and nurses whose content changes according to a daily cycle, including gene products involved in the development of and increasing the response to stimuli in the visual system of foragers; muscle development, including the development of structures involved in muscle contraction and the development of myofilaments in nurses; and the generation of precursor metabolites and the energy in the two groups of workers. In addition, transcription of the genes encoding P-450 daily rhythm occurs in both foragers and nurses.

The rhythm of resistance against insecticides is a result of diurnal and seasonal changes in gene expression related to the detoxification system, for which the pattern of expression depends on the time of day when honeybee workers are foraging [32]. The phenomenon of cyclic changes in the susceptibility against insecticides in the case of honeybees correlates with the time of most intensive secreting nectar by flowering plants in a given season, as well as the ambient temperature enabling flights to seek food [33]. The phenomenon of cyclic changes in honeybee susceptibility to pesticides also was observed in our study. It is possible that the nectar secreted by plants or temperature changes during the day are factors responsible for differences in the susceptibility $A$. mellifera to xenobiotics that depends on the time of year as observed in our study.

There is a significant correlation between the time of day, the season of intoxication, and the toxicity of applied preparations. Our results indicate that the higher the bees' operational readiness to achieve food (but not the work itself, because the methodology used in our study almost prevented bees from demonstrating increased physical activity) that is seen during the day and determined by the cyclic activity of biological clocks [29], the stronger the toxic effect of chemical stressors. As indicated by Xu et al. [32] and Lamia et al. [34], time for foraging is one of the most important zeitgebers in animal organisms. This fact has been additionally confirmed by tests carried out on young worker bees in which some of the cyclic phenomena characteristic of older worker bees were not observed [35]. Other reactions of this type, such as the rhythmic changes of the susceptibility to insecticides [24] in young bees, are not so clearly visible as in older individuals [25]. Our results coincide with the assumptions of Eesa and Cutcomp [36], who, after analyzing many publications concerning the chronotoxicology of insects, concluded that in most cases the toxins have a stronger effect on animals in the daily phase of activity than during the resting phase. Due to the increased activity of animals, the intensified metabolism contributes to a faster absorption and spread of the toxins.

On the other hand, different results were obtained by Onyeocha and Fuzeau-Braesch [37] and Pszczółkowski and Dobrowolski [23]. These investigators suggested that the highest activity of the detoxification system coincided with the time of the acquisition of food. However, it should be taken into account that the bees also used the stocks of food stored in the hive, therefore the period of foraging was not necessarily coincident closely with the time of feeding.

Bees, which are genetically incapable of pesticide detoxifying, are unique in the world of insects, whose resistance to plant protection chemicals has become the rule rather than the exception. Compared to other insects, the honeybee genome has just 46 genes responsible for cytochrome $\mathrm{p} 450$ functioning, unlike other insects, which have 80 or more [38], and it contains less than $20 \%$ of genes of the CYP4 clade, which is strongly associated with pyrethroid resistance in other species [39].

Knowledge about the impact of circadian phenomena on the susceptibility of the honeybees to biocides can be used to reduce the losses in the number of bee families in agricultural areas, especially when the use of crop protection formulations also takes place with a certain cyclicity [40].

We have found that all preparations applied during the light phase of the day were more toxic to worker bees than the same preparations used at night. During the spring, episodes of daily maximum and minimum honeybee susceptibility to insecticides occurred about two to four hours later than in the summer. It can be expected that cyclical succession of seasons of the year also can affect the diurnal rhythm of regulatory mechanisms in the honeybee body. It is likely that the season of the year is decisive here, especially because in a study on behaviour of the worker honeybees in a thermal gradient chamber Grodzicki and Caputa [41] showed that the rhythm of ambient temperature selection is different between spring 
and summer, which is linked to the annual rhythm of honeybee colony development. Nevertheless, the studied insecticides, even when applied at significantly lower doses than those recommended to control pests on crops, were always characterized by high toxicity to worker bees.

As indicated above, the 24-hour rhythms may determine the force of the biocide action. However, it is known that biocides can also modify the rhythmicity by themselves [42]. In the case of worker bees this may mean that sub-lethal doses of substances could break down the time of day in which bees usually fly (flight times do not match the peak of the nectaring period). Hence, bees that forage in abnormal times of the day may have a higher risk of exposure to the biocides applied in the evenings.

\section{Conclusions}

1. Pyrethroid insecticides, in doses applied, appeared to be toxic to worker bees $A$. mellifera.

2. Worker bees $A$. mellifera were more susceptible to pyrethroid action during the day than at night.

3. In summer, the time of day of the highest susceptibility of worker bees to pyrethroid action occurred several hours earlier than in spring.

\section{References}

1. BACANDRITSOS N., GRANATO A., BUDGE G., PAPANASTASIOU I., ROINIOTI E., CALDON M., FALCARO C., GALLINA A., MUTINELLI F. Sudden deaths and colony population decline in Greek honey bee colonies. J. Invertebr. Pathol. 105, 335, 2010.

2. BONNING B.C. The dicistroviridae: an emerging family of invertebrate viruses. ViroSin. 24 (5), 415, 2009.

3. JOHNSON R.M., EVANS J.D., ROBINSON G.E., BERENBAUM M.R. Changes in transcript abundance relating to colony Collapse Disorder in honey bees (Apis mellifera). Proc. Natl. Acad. Sci. U. S. A. 106, 14790, 2009.

4. NAUG D. Nutritional stress due to habitat loss may explain recent honeybee colony collapses. Biol. Conserv. 142 2369, 2009.

5. STINDL R., STINDL W. Vanishing honeybees: is the dying of adult worker bees a consequence of short telomeres and premature aging? Med. Hypotheses. 75, 387, 2010.

6. CORE A., RUNCKEL C., IVERS J., QUOCK C., SIAPNO T., DENAULT S., BROWN, DERISI J., SMITCH C. D., HEFERNIK J. A new threat to honey bees, the parasitic phorid fly Apocephalus borealis. Plos ONE. 7 (1), e29639, 2012.

7. PAXTON R.J. Does infection by Nosema ceranae cause "Colony Collapse Disorder" in honey bees (Apis mellifera)? J. Apic. Res. 49 (1), 80, 2010.

8. DESNEUX N., DECOURTYE A., DELPUECH J.M. The sublethal effects of pesticides on beneficial arthropods. Annu. Rev. Entomol. 52, 81, 2007.

9. FRANCO A.O, GOMES M.G.M., ROWLAND M., COLEMAN P.G., DAVIES C.R. Controlling malaria using livestock-based interventions: a one health approach. PLoS ONE. 9 (7), 01699, 2014.
10. RANSON H., GUESSAN R.N., LINES L., MOIROUX N., NKUNI Z., CORBEL V. Pyrethroid resistance in African anopheline mosquitoes: what are the implications for malaria control? Trends Parasitol. 27 (2), 91, 2011.

11. CORBEL V., AKOGBETO M., DAMIEN G.B., DJENONTIN A., CHANDRE F., ROGIER C., MOIROUX N., CHABI J., BANGANNA B., PADONOU G.G., HENRY M.-C. Combination of malaria vector control interventions in pyrethroid resistance area in Benin: a cluster randomised controlled trial. The Lancet. 12 (8), 617, 2012.

12. ANADÓN A., MARTÍNEZ-LARRAÑAGA M.R., MARTÍNEZ M.A. Use and abuse of pyrethrins and synthetic pyrethroids in veterinary medicine. Vet. J. 182 (1), 7, 2009.

13. GEORGE D.R., FINN R.D., GRAHAM K.M., SPARAGANO O.A.E. Present and future potential of plantderived products to control arthropods of veterinary and medical significance. Parasit. Vectors. 7, 28, 2014.

14. DEWAILLY E., FORDE M., ROBERTSON L., KADDAR N., LAOUAN SIDI EA., CÔTÉ S., GAUDREAU E., DRESCHER O., AYOTTE P. Evaluation of pyrethroid exposures in pregnant women from 10 Caribbean countries. Environ Int. 63, 201, 2014.

15. TRUNNELLE K., BENNETT D., TULVE N., CLIFTON M., DAVIS M., CALAFAT A., MORAN R., TANCREDI D., HERTZ-PICCIOTTO I. Urinary pyrethroid and chlorpyrifos metabolite concentraitons in northern California families and their relationship to indoor home insecticide levels, part of the study of use of products and exposure related behavior (SUPERB). Environ. Health Perspect. 48 (3), 1931, 2014.

16. YAN H., QIAO F., TIAN M., ROW K.H. Simultaneous determination of nine pyrethroids in indoor insecticide products by capillary gas chromatography. J. Pharm. Biomed. Anal. 51 (3), 774, 2010.

17. ORUC H.H., HRANITZ J.M., SORUCU A., DUELL M., CAKMAK I., AYDIN L., ORMAN A. Determination of acute oral toxicity of flumethrin in honey bees. J. Econ. Entomol. 105 (6), 1890, 2012.

18. ZHOU T., ZHOU W., WANG Q., DAI P.L., LIU F., ZHANG Y.L., SUN J.H. Effects of pyrethroids on neuronal excitability of adult honeybees Apis mellifera. Pestic. Biochem. Physiol. 100, 35, 2011.

19. BRECKENRIDGE C.B., HOLDEN L., STURGESS N., WEINER M., SHEETS L., SARGENT D., SODERLUND D.M., CHOI J.S., SYMINGTON S., CLARK J.M., BURR S., RAY D. Evidence for a separate mechanism of toxicity for the Type I and the Type II pyrethroid insecticides. NeuroToxicol. 30 (1), 17, 2009.

20. WANG S.-Y., WANG G.K. Voltage-gated sodium channels as primary targets of diverse lipid-solube neurotoxins. Cell. Signal. 15, 151, 2003.

21. SODERLUND D., CLARK J.M., SHEETS L. P., MULLIN L.S., PICCIRILlO V.J., SARGENT D., STEVENS J.T., WEINER M.L. Mechanism of pyrethroid neurotoxicity: implications for cumulative risk assessment. Toxicol. 171, 3, 2002.

22. MINEAU P., HARDING K.M., WHITESIDE M., FLETCHER M.R., GARTHWAITE D., KNOPPER I.D. Using reports of bee mortality in the field to calibrate laboratory-derived pesticide risk indices. Environ. Entomol. 37 (2), 546, 2008

23. PSZCZOLKOWSKI M.A., DOBROWOLSKI M. Circadian dynamics of locomotor activity and deltamethrin susceptibility in the pine weevil, Hylobius abietis. Phytoparasitica 27 (1), 19, 1999.

24. PIECHOWICZ B., STAWARCZYK K., STAWARCZYK M. Circadian changes in susceptibility of young honeybee 
workers to intoxication by pyrethroid, carbamate, organophosphorus, benzoyl urea and pyridine derivative insecticides. J. Plant Prot. Res. 52 (2), 286, 2012.

25. PIECHOWICZ B., GRODZICKI P., STAWARCZYK K., STAWARCZYK M. Circadian and seasonal changes in the honeybee (Apis mellifera) workers susceptibility to diazinon, teflubenzuron, pirimicarb and indoxacarb. Pol. J. Environ. Stud. 22 (5), 1457, 2013.

26. PIECHOWICZ B., GRODZICKI P. Circadian changes in susceptibility of various species of Gryllidae to insecticides, depending on time of intoxication and size of tested group. Pol. J. Environ. Stud. 23 (5), 1861, 2014.

27. PIECHOWICZ B., GRODZICKI P. Effect of temperature on toxicity of selected insecticides to forest beetle Anoplotrupes stercorosus. Chem. Didact. Ecol. Metrol. 18 (1-2), 103, 2014.

28. RODRIGUEZ-ZAS S.L., SOUTHEY B.R. , SHEMESH Y., RUBIN E.B., COHEN M., ROBINSON G.E., BLOCH G. Microarray analysis of natural socially regulated plasticity in circadian rhythms of honey bees. J. Biol. Rhythms. 27 (1), $12,2012$.

29. BLOCH G. The social clock of the honeybee. J Biol Rhythms. 25 (5), 307, 2010.

30. STABENTHEINER A., KOVAC H., BRODSCHNEIDER R. Honeybee colony thermoregulation - regulatory mechanisms and contribution of individuals in dependence on age, location and thermal stress. PLoS ONE. 5 (1), e8967, 2010.

31. MOORE D. Honey bee circadian clocks: behavioral control from individual workers to whole-colony rhythms. J. Insect. Physiol. 47, 843, 2001.

32. XU K., DIANGELO J.R., HUGHES M.E., HOGENESCH J.B., SEHGAL A. The circadian clock interacts with metabolic physiology to influence reproductive fitness. Cell Metab. 13 (6), 639, 2011.

33. ABOU-SHAARA H.F. The foraging behaviour of honey bees, Apis mellifera: a review. Vet. Med. 59 (1), 1, 2014.
34. LAMIA K.A., STORCH K.F., WEITZ C.J. Physiological significance of a peripheral tissue circadian clock. Proc. Natl. Acad. Sci. USA. 105 (39), 15172, 2008.

35. NIJLAND M.J.M., HEPBURN H.R. Ontogeny of a circadian rhythm in the cluster temperature of honeybees. S. Afr. J. Sci. 81, 100, 1985.

36. EESA N.M., CUTKOMP L.K. Pesticide chronotoxicity to insects and mites: an overview. J. Islam. Acad. Sci. 8 (1), 21, 1995

37. ONYEOCHA F.A., FUZEAU-BRAESCH S. Circadian rhythm changes in toxicity of the insecticide dieldrin on larvae of the migratory locust Locusta migratoria migratoroides. Chronobiol. Int. 8 (2), 103, 1991.

38. CLAUDIANOS C., RANSON H., JOHNSON R. M., BISWAS S., SCHULER M.A., BERENBAUM M.R., FEYEREISEN R., OAKESHOTT J.G. A deficit of detoxification enzymes: pesticide sensitivity and environmental response in the honeybee. Insect Mol. Biol. 15 (5), 615, 2006.

39. THE HONEYBEE GENOME SEQUENCING CONSORTIUM. Insights into social insects from the genome of the honeybee Apis mellifera. Nature. 443, 931, 2006.

40. LAMBERT O., PIROUX M., PUYO S., THORIN C., L'HOSTIS M., WIEST L., BULETE A., DELBAC F., POULIQUEN H. Widespread occurrence of chemical residues in beehive matrices from apiaries located in different landscapes of western France. Plos ONE. 8 (6), e67007, 2013.

41. GRODZICKI P., CAPUTA M. Diurnal and seasonal changes in thermal preference of single, isolated bees and small groups of bees (Apis mellifera L.). J. Insect Behav. 27 (4), 701, 2014.

42. TEGOWSKA E. Insecticides and thermoregulation of insects. Pestycydy/Pesticides. 1 (4), 47, 2003. 